\title{
HIGH-RESOLUTION OPTICAL SPECTROSCOPY OF DY Cen: DIFFUSE INTERSTELLAR BANDS IN A PROTO-FULLERENE CIRCUMSTELLAR ENVIRONMENT?
}

\author{
D. A. García-Hernández ${ }^{1,2}$, N. Kameswara RaO ${ }^{3,4,5}$, and David L. Lambert ${ }^{5}$ \\ ${ }^{1}$ Instituto de Astrofísica de Canarias, C/Via Láctea s/n, E-38200 La Laguna, Spain; agarcia@iac.es \\ ${ }^{2}$ Departamento de Astrofísica, Universidad de La Laguna (ULL), E-38206 La Laguna, Spain \\ 3 543, 17th Main, IV Sector, HSR Layout, Bangalore 560102, India; nkrao@iiap.res.in \\ ${ }^{4}$ Indian Institute of Astrophysics, Bangalore 560034, India \\ ${ }^{5}$ The W.J. McDonald Observatory, University of Texas, Austin, TX 78712-1083, USA; dll@ astro.as.utexas.edu \\ Received 2012 August 31; accepted 2012 October 3; published 2012 October 16
}

\begin{abstract}
We search high-resolution and high-quality VLT/UVES optical spectra of the hot R Coronae Borealis star DY Cen for electronic transitions of the $\mathrm{C}_{60}$ molecule and diffuse interstellar bands (DIBs). We report the non-detection of the strongest $\mathrm{C}_{60}$ electronic transitions (e.g., those at $\sim 3760,3980$, and $4024 \AA$ ). The absence of $\mathrm{C}_{60}$ absorption bands may support recent laboratory results, which show that the $\sim 7.0,8.5,17.4$, and $18.8 \mu \mathrm{m}$ emission features seen in DY Cen - and other similar objects with polycyclic-aromatic-hydrocarbon-like dominated IR spectra—are attributable to proto-fullerenes or fullerene precursors rather than to $\mathrm{C}_{60}$. DIBs toward DY Cen are normal for its reddening; the only exception is the DIB at $6284 \AA$ (possibly also the $7223 \AA$ DIB) which is found to be unusually strong. We also report the detection of a new broad (FWHM $\sim 2 \AA$ ) and unidentified feature centered at $\sim 4000 \AA$. We suggest that this new band may be related to the circumstellar proto-fullerenes seen at infrared wavelengths.
\end{abstract}

Key words: astrochemistry - circumstellar matter - dust, extinction - ISM: molecules - stars: chemically peculiar - stars: individual (DY Cen)

Online-only material: color figures

\section{INTRODUCTION}

Fullerenes and fullerene-related molecules have attracted much attention since their discovery in the laboratory (Kroto et al. 1985) due to their potential applications in superconducting materials, optical devices, and medicine. In the astrophysical context, these complex molecules have been proposed as explanations for unidentified astronomical features such as the intense UV absorption band at $217 \mathrm{~nm}$ (e.g., Cataldo \& IglesiasGroth 2009) and the enigmatic diffuse interstellar bands (DIBs; Herbig 1995, 2000; Iglesias-Groth 2007). The remarkable stability of fullerenes against intense radiation (e.g., Cataldo et al. 2009) has encouraged the idea that fullerenes should be present in the interstellar medium (ISM). Indeed, the 9577 and $9632 \AA$ DIBs observed in a few reddened stars lie near two electronic transitions of the $\mathrm{C}_{60}$ cation observed in rare gas matrices (Foing \& Ehrenfreund 1994), but the presence of fullerenes in astrophysical environments has been uncertain until recently, when the mid-IR spectral signatures of the $\mathrm{C}_{60}$ and $\mathrm{C}_{70}$ fullerenes were detected in several planetary nebulae (PNe; Cami et al. 2010; García-Hernández et al. 2010, 2011a).

Interestingly, García-Hernández et al. (2010) showed that contrary to theoretical and experimental expectation, fullerenes are efficiently formed in normal H-rich circumstellar environments. The prevailing laboratory view was that the synthesis of fullerenes is much more efficient under hydrogen-poor conditions (Kroto et al. 1985; De Vries et al. 1993). Thus, circumstellar envelopes around R Coronae Borealis (RCB) stars (see, e.g., Lambert \& Rao 1994 for a review) have been considered promising environments for the formation of fullerene molecules (Goeres \& Sedlmayr 1992). This is because the RCBs' hydrogen deficiency together with the He- and C-rich character of the gas resembles the laboratory conditions where fullerenes are produced. However, this suggestion is not supported by our recent Spitzer observations of a large sample of RCB stars, which show that fullerene formation is inefficient in the highly H-deficient environments characteristic of most RCBs (García-Hernández et al. 2011b, 2011c).

Surprisingly, the only exception among RCBs is that the least H-deficient RCB star DY Cen (possibly also the second least $\mathrm{H}$-deficient RCB star V854 Cen), which shows mid-IR emission features at $\sim 7.0,8.5,17.4$, and $18.8 \mu \mathrm{m}$, is coincident with the IR transitions of $\mathrm{C}_{60}$. Contrary to fullerene-containing $\mathrm{PNe}$, the latter $\mathrm{C}_{60}$ mid-IR features in DY Cen are seen in conjunction with very strong polycyclic aromatic hydrocarbon (PAH) features. This striking difference between the IR spectra of fullerene-containing PNe and DY Cen is explained by Duley \& Hu (2012) who attribute the $\sim 7.0,8.5,17.4$, and $18.8 \mu \mathrm{m}$ features in DY Cen to proto-fullerenes (or fullerene precursors) rather than to the $\mathrm{C}_{60}$ molecule. Based on their laboratory spectroscopy of hydrogenated amorphous carbon (HAC) nanoparticles, Duley \& Hu (2012) suggest that these four IR features may be due to proto-fullerenes in sources such as reflection nebulae, RCB stars, and proto-PNe, which also show the unidentified $16.4 \mu \mathrm{m}$ feature and other very strong PAH-like features. In light of the now suspect infrared identification of neutral $\mathrm{C}_{60}$, we sought to detect the molecule's electronic transitions in absorption from DY Cen's optical spectrum, as an additional check to the presence of $\mathrm{C}_{60}$ and see whether Duley \& Hu's (2012) "proto-fullerenes" suggestion could be substantiated.

\section{VLT/UVES OPTICAL SPECTROSCOPY}

High-resolution $(R \sim 30,000)$ and high-quality (signal-tonoise ratio $(\mathrm{S} / \mathrm{N}) \geqslant 200)$ optical $(\sim 3300-9450 \AA)$ spectroscopic observations of DY Cen $(V=12.7, E(B-V)=0.50$; see Table 1) were obtained in the period 2010 February-March at the European Southern Observatory (ESO). The UVES spectrograph at the ESO VLT (Paranal, Chile) was used with the 

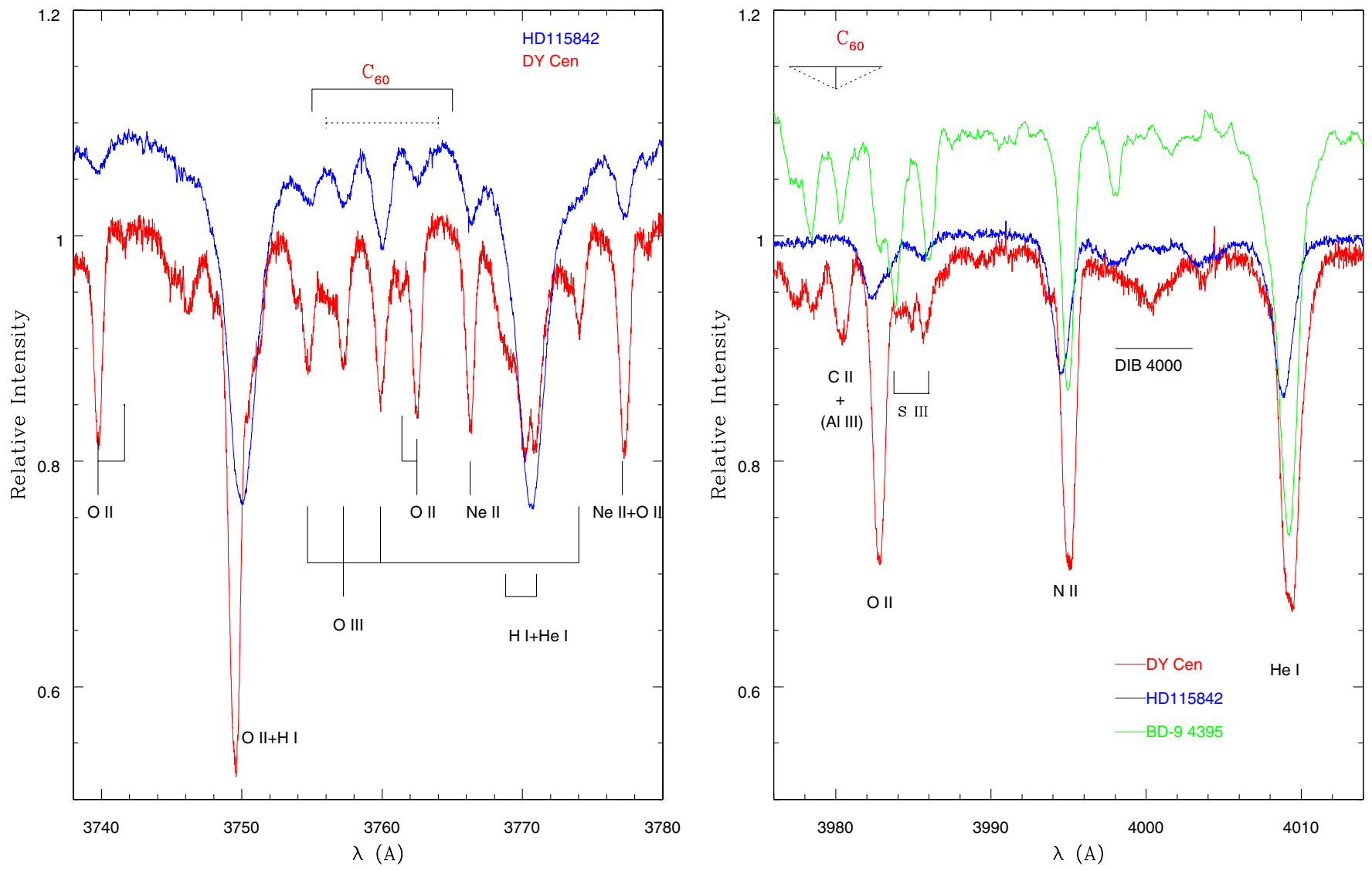

Figure 1. Spectra of DY Cen (in red) and HD 115842 (in blue) around $3760 \AA$ (left panel) and $4000 \AA$ (right panel; where the eHe star BD - $9^{\circ} 4395$ is also displayed in green) are shown. The expected positions of the $\mathrm{C}_{60}$ features are marked on top of the spectra. The FWHM of the features is indicated. Note that there is no evidence (additional absorption) in DY Cen for the presence of the neutral $\mathrm{C}_{60}$ features at 3760 and $3980 \AA$. . However, there is an additional absorption band at $4000 \AA$ in DY Cen (marked by a dark line and written DIB4000), which is not present in either HD 115842 or in the EHe star BD $-9^{\circ} 4395$ that is expected to show similar spectrum as DY Cen.

(A color version of this figure is available in the online journal.)

Table 1

Observational Parameters of DY Cen

\begin{tabular}{lccccccccc}
\hline \hline Star & $l$ & $b$ & $V$ & $B-V$ & $E(B-V)$ & Sp. Type & $M_{\mathrm{V}}$ & $\begin{array}{c}\text { Distance } \\
(\mathrm{pc})\end{array}$ & $\begin{array}{c}\text { Ref } \\
\text { DY Cen }\end{array}$ \\
& 307.958 & 8.293 & 12.7 & 0.33 & $0.47-0.50$ & B0 Ia & & 4800 & 1 \\
HD115842 & 307.080 & 6.834 & 6.04 & 0.30 & 0.50 & B0.5 Ia & -8.0 & 3581 & 2 \\
& & & & & & & -6.9 & 2157 & 2 \\
\hline
\end{tabular}

References. (1) De Marco et al. 2002; (2) Krelowski et al. 2010; Hunter et al. 2006.

1".2 slit and the standard setting DIC2 (390+760). We obtained 11 individual exposures of $1800 \mathrm{~s}$ each, giving a total exposure time of $5.5 \mathrm{hr}$. The $\mathrm{S} / \mathrm{N}$ in the continuum in the summed DY Cen's spectrum is $\sim 200$ at $4000 \AA$ and higher than 250 at wavelengths longer than $6000 \AA$. The observed spectraprocessed by the UVES data reduction pipeline (Ballester et al. 2000)—were corrected for heliocentric motion and the stellar continuum was fitted by using standard astronomical tasks in IRAF.

As a comparison star for DY Cen in our search for $\mathrm{C}_{60}$ and DIBs, we selected the very nearby B 0.5 Ia bright supergiant HD 115842 , which only differs in Galactic longitude and latitude by 0.88 and 1.46 , respectively. This is because HD 115842 has the same reddening $(E(B-V)=0.5)$ as DY Cen and it may be located at a comparable distance, sampling similar ISM conditions. A comparison between HD 115842 and DY Cen will probably enlighten the environmental changes associated with DY Cen. Thus, HD 115842 was also observed on the same dates as DY Cen with the same VLT/UVES setup. Table 1 compares DY Cen with the comparison star HD 115842 in terms of Galactic coordinates, spectral type, magnitude, and reddening. Finally, a spectrum of $\mathrm{BD}-9^{\circ} 4395$, an extreme helium (EHe) star that shows a photospheric spectrum similar to that of DY Cen, was obtained by us with the $2.7 \mathrm{~m}$ Harlan Smith telescope and the Tull coudé spectrograph (Tull et al. 1995) at a spectral resolving power of 60,000.

\section{A SEARCH FOR NEUTRAL $\mathrm{C}_{60}$}

We have inspected the optical spectrum of DY Cen in order to search for the presence of optical absorptions of neutral $\mathrm{C}_{60}$. The allowed transitions at wavelengths below $4100 \AA$ are much more intense than those of the Herzberg-Teller induced forbidden transitions at longer wavelengths (Leach 1992). 
Table 2

Diffuse Interstellar Bands in DY Cen and HD $115842^{\mathrm{a}}$

\begin{tabular}{|c|c|c|c|c|c|c|c|c|c|}
\hline \multirow{2}{*}{$\begin{array}{l}\text { DY Cen } \\
\lambda_{c} \\
(\AA)\end{array}$} & \multicolumn{9}{|c|}{ HD 115842} \\
\hline & $\begin{array}{c}\text { FWHM } \\
(\AA)\end{array}$ & $\begin{array}{l}\text { EQW } \\
(\mathrm{m \AA})\end{array}$ & $A_{c}$ & $\mathrm{~S} / \mathrm{N}$ & $\begin{array}{l}\lambda_{c} \\
(\AA)\end{array}$ & $\begin{array}{c}\text { FWHM } \\
(\AA)\end{array}$ & $\begin{array}{l}\text { EQW } \\
(\mathrm{m} \AA)\end{array}$ & $A_{c}$ & $\mathrm{~S} / \mathrm{N}$ \\
\hline 4000.38 & 1.92 & 91 & 0.04 & 188 & & & & & 412 \\
\hline 4429.66 & 21.10 & 1034 & 0.05 & 273 & 4428.50 & 23.00 & 932 & 0.04 & 436 \\
\hline 5780.46 & 2.14 & 256 & 0.10 & 209 & 5780.39 & 2.10 & 257 & 0.11 & 307 \\
\hline 5796.92 & 0.91 & 72 & 0.07 & 199 & 5796.90 & 0.89 & 105 & 0.11 & 264 \\
\hline 5849.67 & 0.82 & 32 & 0.02 & 272 & 5849.58 & 0.83 & 43 & 0.05 & 330 \\
\hline 6065.11 & 0.61 & 6 & 0.01 & 312 & 6065.00 & 0.64 & 7 & 0.01 & 635 \\
\hline 6089.62 & 0.53 & 7 & 0.01 & 282 & 6089.59 & 0.53 & 13 & 0.02 & 490 \\
\hline \multirow[t]{2}{*}{6112.7} & 1.00 & 12 & 0.01 & 300 & 6112.97 & 0.62 & 12 & 0.02 & 490 \\
\hline & & & & & 6116.62 & 0.94 & 8 & 0.01 & 490 \\
\hline 6139.75 & 0.82 & 5 & 0.01 & 267 & 6139.72 & 0.59 & 8 & 0.01 & 570 \\
\hline 6195.80 & 0.60 & 27 & 0.04 & 284 & 6195.80 & 0.50 & 33 & 0.06 & 636 \\
\hline 6202.81 & 1.28 & 62 & 0.04 & 336 & 6202.83 & 1.35 & 64 & 0.04 & 636 \\
\hline 6233.79 & 0.79 & 10 & 0.01 & 336 & 6233.80 & 0.66 & 14 & 0.02 & 560 \\
\hline 6269.70 & 1.21 & 36 & 0.02 & 276 & 6269.80 & 1.38 & 70 & 0.04 & 582 \\
\hline 6283.53 & 4.29 & 543 & 0.12 & 276 & 6283.75 & 4.43 & 413 & 0.09 & 582 \\
\hline 6375.97 & 0.92 & 13 & 0.02 & 273 & 6375.91 & 0.90 & 29 & 0.03 & 340 \\
\hline $6379.25^{\mathrm{b}}$ & 1.04 & 53 & & & 6379.04 & 0.64 & 70 & 0.10 & 340 \\
\hline 6597.26 & 0.53 & 8 & 0.01 & 295 & 6597.19 & 0.58 & 9 & 0.01 & 378 \\
\hline 6613.41 & 1.03 & 83 & 0.08 & 295 & 6613.45 & 1.04 & 149 & 0.14 & 304 \\
\hline 6660.60 & 0.64 & 12 & 0.02 & 314 & 6660.53 & 0.80 & 39 & 0.05 & 265 \\
\hline \multicolumn{10}{|l|}{7223.75} \\
\hline 8620.88 & 3.75 & 95 & 0.02 & 106 & 8620.94 & 3.92 & 105 & 0.02 & 243 \\
\hline
\end{tabular}

Notes.

a The $3 \sigma$ errors in the EQWs scale like $\sim 3 \times \mathrm{FWHM} /(\mathrm{S} / \mathrm{N})$ while we estimate that the FHWMs are precise to the $0.03 \AA$ level.

b Blended with N II $6379.98 \AA$.

The strongest allowed electronic transitions of neutral gas phase $\mathrm{C}_{60}$ molecules, as predicted from laboratory experiments, are located at $3760 \pm 5,3980 \pm 0.5$, and $4024.0 \pm 0.5 \AA$ with widths of 8,6 , and $4 \AA$, respectively (Sassara et al. 2001). The oscillator strength $(f)$ of the $\sim 4024 \mathrm{C}_{60}$ band is similar to that at $3980 \AA$ (Leach 1992), while the $3760 \AA$ band has a five times higher oscillator strength (Braga et al. 1991). DY Cen's optical spectrum is dominated by strong $\mathrm{He}_{\mathrm{I}}, \mathrm{C}$ II, Ne I, O II, and $\mathrm{N}$ II absorption and variable emission lines together with nebular forbidden emission lines from [O II], [N II], [S II], etc. The presence of these absorption and emission lines (FWHM $1 \AA$ ) complicates the detection of broad and weak absorption features.

We can find no evidence for the presence of $\mathrm{C}_{60}$ in absorption (or emission) at the wavelengths of the expected electronic transitions:

The $3760 \pm 5 \AA$ band. This, the strongest of the three in our bandpass, falls amongst a series of $\mathrm{O}$ II lines. These lines give consistent $\mathrm{O}$ abundances suggesting that none is blended with a $\mathrm{C}_{60}$ feature (Figure 1, left panel), even though its central wavelength is uncertain by $\pm 5 \AA$ (Sassara et al. 2001). Note that the apparent additional absorption at $3756 \AA$ in DY Cen is probably a stellar blend (Figure 1, left panel).

The $3980 \pm 0.5 \AA$ band. This coincides with a stellar C II line from a multiplet represented by other lines. A secondary contributor may be the Al III $5 d^{2} D-8 f^{2} F^{\circ}$ multiplet (Figure 1, right panel). The $\mathrm{C}$ II lines are present also in $\mathrm{BD}+9^{\circ} 4395$. This coincidence and the line's "stellar" width show that $\mathrm{C}_{60}$ is not a significant contributor. An interesting feature of the right panel of Figure 1 is the appearance of an unidentified broad feature in DY Cen at $4000 \AA$ (FWHM $\sim 2 \AA$; equivalent width (EQW) of $91 \mathrm{~m} \AA$ ). It is to be noted here that the $4000 \AA$ feature is real. First, a strong argument in favor of the $4000 \AA$ feature being real is that it does not appear in the spectra of the comparison star taken with the same setup and at the same time. Second, the $4000 \AA$ band is clearly seen when reducing the corresponding Echelle order alone. This is not a known DIB; it is not present in HD 115842 where all DIBs have a similar strength to those in DY Cen (see below) and neither is it listed by Hobbs et al. (2008). Identification as $\mathrm{C}_{60}$ would demand a wavelength error of either $20 \AA$ for the $3980 \AA$ transition or $24 \AA$ for the $4024 \AA$ transition but Sassara et al. give the wavelength uncertainty as just $\pm 0.5 \AA$ for both transitions.

The $C_{60}$ band at $4024.0 \pm 0.5$. This is irretrievably blended with the He I $4026 \AA$ line and adjacent lines.

We estimate that the $1 \sigma$ detection limits on the column density from the 3760 and $3980 \AA \mathrm{C}_{60}$ bands in our DY Cen spectra $(\mathrm{S} / \mathrm{N} \sim 200)$ are $4.6 \times 10^{12}$ and $1.5 \times 10^{13} \mathrm{~cm}^{-2}$, respectively. ${ }^{6}$ It is of interest to compare this column density limit of about $10^{13} \mathrm{~cm}^{-2}$ with the number of $\mathrm{C}_{60}$ molecules calculated from the infrared emission features attributed to these molecules. This exercise is attempted next.

The IR features measure the number of molecules in excited vibrational states but the electronic transitions sought here depend on the molecules in their ground vibrational state. Analysis of the strengths of the IR features with the estimated $A$-values (Cami et al. 2010; García-Hernández et al. 2010, $2011 b$ ) shows that the excitation temperature is about $600 \mathrm{~K}$. With this temperature, we estimate the number of molecules in the ground state: $N\left(\mathrm{C}_{60}\right)=1.0 \times 10^{46} d^{2}$ where $d$, the distance to DY Cen, is given in kpc.

The column density pertinent to our non-detection of $\mathrm{C}_{60}$ depends on how the line of sight to the star intersects the

\footnotetext{
$61 \sigma$ detection limits for the EQWs in our spectra scale as $\sim 1.064 \times$ FWHM/(S/N) (see, e.g., Hobbs et al. 2008).
} 


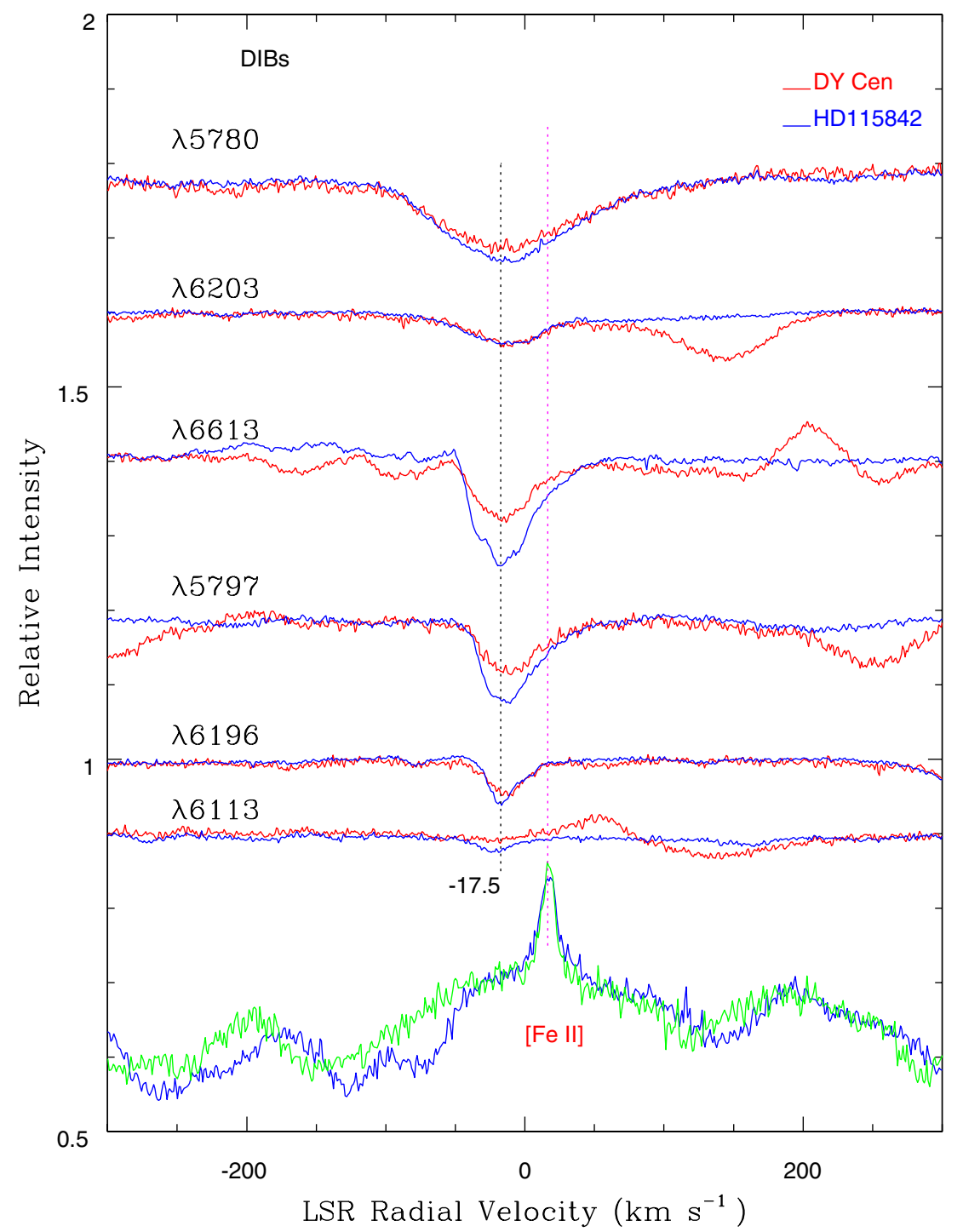

Figure 2. Profiles of six DIBs of various strengths and widths are plotted with respect to the LSR radial velocity. Note that the profiles are shifted vertically for clarity. The [Fe II] profile at the bottom shows the DY Cen's systemic radial velocity. Note that the strengths and shapes of 5780 and $6203 \AA$ DIBs are almost identical for both stars. The other profiles show that DIBs toward the DY Cen sight of line are weaker than toward HD 115842. To convert the velocity scale to heliocentric, 4.6 and $5.1 \mathrm{~km} \mathrm{~s}^{-1}$ should be added to the LSR velocities of DY Cen and HD 115842, respectively.

(A color version of this figure is available in the online journal.)

$\mathrm{C}_{60}$-containing part of the circumstellar shell. Assume a spherical shell centered on the star with inner and outer radii of $R_{\text {out }}$ and $R_{\mathrm{in}}$, respectively, with the line of sight running directly from $R_{\text {out }}$ to $R_{\text {in }}$. Assume a uniform density of $\mathrm{C}_{60}$ molecules throughout the shell, then the density of molecules is $n\left(\mathrm{C}_{60}\right)$, where

$$
n\left(\mathrm{C}_{60}\right)=\frac{7.0 \times 10^{-41}}{R_{\mathrm{out}}^{3}-R_{\mathrm{in}}^{3}} N\left(\mathrm{C}_{60}\right),
$$

where $R_{\text {out }}$ and $R_{\text {in }}$ are given in AU.

Shell radii are estimated from the run of dust temperature with distance from the star. The equilibrium temperature of a gray dust grain $T_{d}(R)$ in an optically thin circumstellar environment is given by

$$
T_{d}(R)=\left(\frac{R_{*}}{2 R}\right)^{0.5} T_{*},
$$

where $R_{*}$ is the stellar radius, $R$ is the radial distance from the stellar center, and $T_{*}$ is the stellar blackbody temperature (Kwok 2007, p. 314, Equation (10.32)). If stellar radii and temperature are estimated from the stellar luminosity $L / L_{\odot}$, the distance $R$ is provided by

$$
R=\frac{1}{430}\left(\frac{L}{L_{\odot}}\right)^{0.5}\left(\frac{T_{\odot}}{T_{d}(R)}\right)^{2}
$$

for $R$ in $\mathrm{AU}$.

Adopting $L / L_{\odot}=10^{4}$, we find $T_{d}=600 \mathrm{~K}$ at $R=21 \mathrm{AU}$ and $T_{d}=270 \mathrm{~K}$ at $104 \mathrm{AU}$ where the former temperature is the excitation temperature of the $\mathrm{C}_{60}$ molecules and the latter temperature is the blackbody temperature of the dust. If we adopt $R_{\text {in }} \simeq 10 \mathrm{AU}$ and $R_{\text {out }} \simeq 100 \mathrm{AU}$, we obtain $n\left(\mathrm{C}_{60}\right)=$ $0.7 d^{2} \mathrm{~cm}^{-3}$ or $n\left(\mathrm{C}_{60}\right)=34 \mathrm{~cm}^{-3}$ for $d=7 \mathrm{kpc}$ and $17 \mathrm{~cm}^{-3}$ for $d=5 \mathrm{kpc}$. Then, the estimates for the $\mathrm{C}_{60}$ column density along the path $\left(R_{\text {out }}-R_{\text {in }}\right)$ are $4.6 \times 10^{16} \mathrm{~cm}^{-2}$ for $7 \mathrm{kpc}$ and $2.3 \times 10^{16} \mathrm{~cm}^{-2}$ for $5 \mathrm{kpc}$.

Such estimates are uncomfortably greater than the observed upper limit of around $10^{13} \mathrm{~cm}^{-2}$. One possible explanation of this 1000-fold discrepancy is that the line of sight to DY Cen does not intersect the $\mathrm{C}_{60}$-containing regions of the 

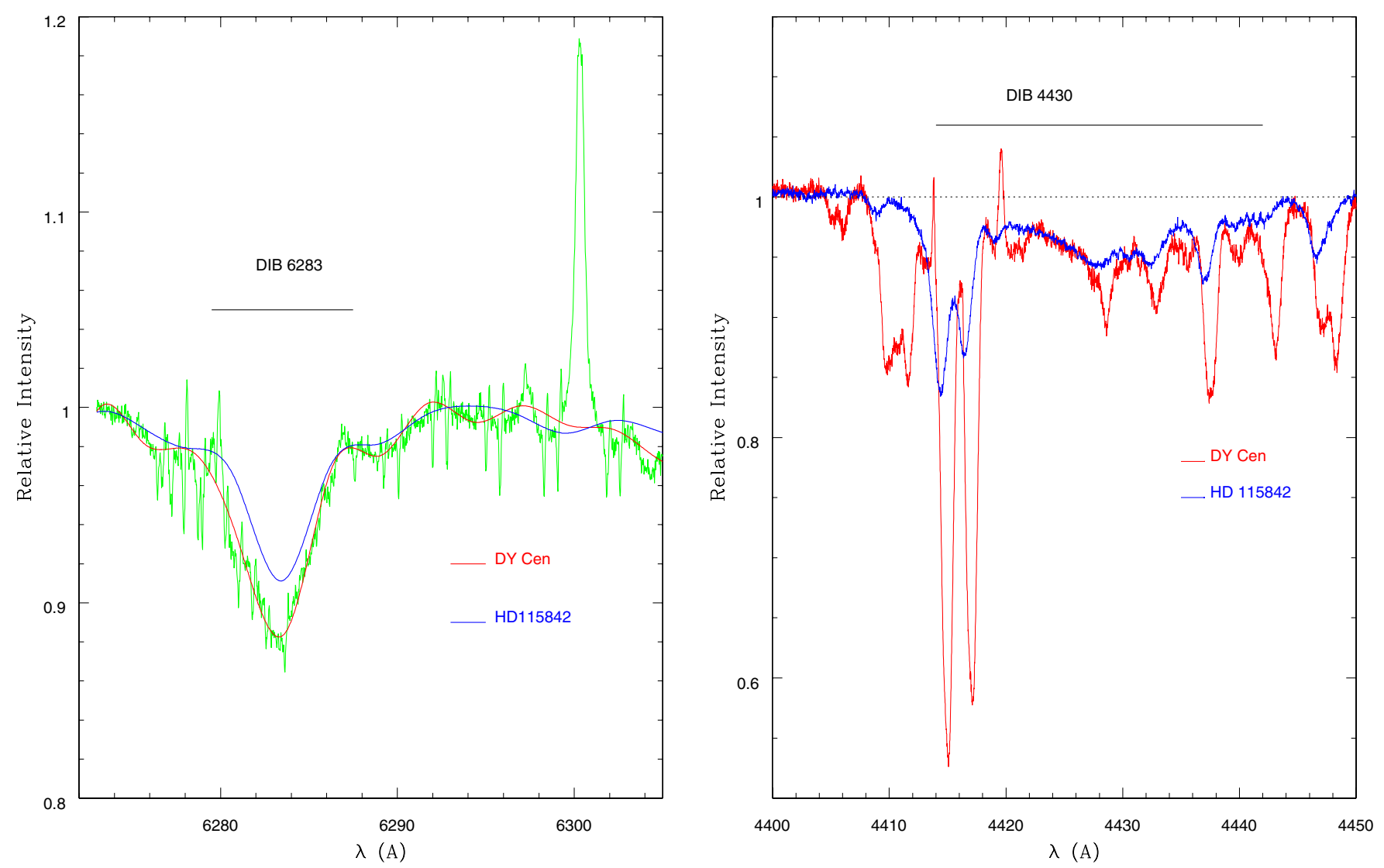

Figure 3. Spectral regions around the DIBs at $6284 \AA$ (left panel) and $4430 \AA$ (right panel) in DY Cen (in red) and HD 115842 (in blue). The telluric line corrected spectrum of both stars are shown by smooth lines (red and blue). The not fully telluric corrected spectrum with emission of [O I] line in DY Cen is shown in green. It is obvious that the $6284 \AA$ DIB is stronger in DY Cen spectrum than toward HD 115842 while the DIB at $4430 \AA$ (right panel) is of similar strength in both stars.

(A color version of this figure is available in the online journal.)

circumstellar envelope. An alternative view raises doubts about the IR detection of the $\mathrm{C}_{60}$ molecule in DY Cen (possibly also in V854 Cen; García-Hernández et al. 2011b), supporting the recent claim that the $\sim 7.0,8.5,17.4$, and $18.8 \mu \mathrm{m}$ features seen in astronomical sources with PAH-like-dominated IR spectra should be attributed to proto-fullerenes or fullerene precursors rather than to $\mathrm{C}_{60}$ (Duley \& $\mathrm{Hu} 2012$ ). The latter seems to be consistent with the characteristics of the DIBs toward DY Cen, which are studied below.

\section{DIFFUSE BANDS FROM FULLERENE PRECURSORS?}

As we have mentioned above, we detect in DY Cen a broad unidentified feature centered at $\sim 4000 \AA$, which is seen in DY Cen only (Figure 1). Note that no DIBs are known at this wavelength (see, e.g., Hobbs et al. 2008). To our knowledge, no molecule is known to exhibit a strong electronic transition at $\sim 4000 \AA$ and this is corroborated by inspecting the NIST Chemistry WebBook Database. ${ }^{7}$ We speculate that this $4000 \AA$ band may be related to the proto-fullerenes or fullerene precursors seen in the IR and that could be very abundant in the circumstellar envelope of DY Cen. Thus, the $4000 \AA$ feature may represent a new and very unusual diffuse band (DB).

We used the exhaustive list of DIBs provided by Hobbs et al. (2008) in HD 204827 to search for them in the spectrum of DY Cen. DIBs detected in DY Cen (and the comparison star

\footnotetext{
7 See http://webbook.nist.gov/chemistry/.
}

HD 115842) are given in Table 2, where we give the central wavelength (after applying the heliocentric correction), FWHM in $\AA$, EQW in $\mathrm{m} \AA$, central depth $\left(A_{c}\right)$, and the $\mathrm{S} / \mathrm{N}$ in the neighboring continuum. Figure 2 compares a selection of DIBs in DY Cen with those in HD 115842. Table 2 (also Figure 2) shows that the DIBs toward DY Cen have about the same or slightly less (up to 30\%) strength, the same FWHM, and a similar radial velocity as those toward HD 115842 . Since both stars have roughly the same interstellar reddening $E(B-V)$, the similar profiles and strengths for their DIBs are not surprising. In addition, for the well-studied DIBs at 5780, 5797, 5850, 6196,6379 , and $6614 \AA$, the normalized equivalent widths $\mathrm{EQW} / E(B-V)$ in DY Cen agree very well with those measured in field stars by Luna et al. (2008) and in the prototype star HD 183143 (Herbig 1995).

The exceptions to the above trends are the DIBs at $6284 \AA$ and at $7223 \AA$. The $7223 \AA$ DIB is stronger in DY Cen than in HD 115824 as seen from the divided spectrum, but we could not estimate its total absorption because of the interference from the superposed telluric lines. Figure 3 (left panel) shows the region of $6284 \AA$ for both stars. The spectra have been cleaned of telluric lines. It is clear that this DIB toward DY Cen is stronger than toward HD 115842, suggesting that the carrier of the $6284 \AA$ DIB (along with $7223 \AA$ ) is different from the rest of the classical DIBs. Indeed, the strength of this DIB seems to be poorly correlated with the interstellar reddening. For example, Luna et al. (2008) and Herbig (1995) obtained $\mathrm{EQW} / E(B-V)$ of 0.90 and 1.50 for field reddened stars and 
HD 183143, respectively, while we measure a value of 1.10 toward DY Cen; Hobbs et al. (2008) give a value of 0.41 in HD 204827. Curiously, the post-asymptotic giant branch star IRAS 06530-0213, which shows the strongest unidentified $21 \mu \mathrm{m}$ feature (Zhang et al. 2010), also shows an unusually strong $6284 \AA$ DIB (see Luna et al. 2008). The $21 \mu \mathrm{m}$ feature may be attributed to HACs or their decomposition products such as fullerene precursors or intermediate products (see, e.g., García-Hernández 2012). It is to be noted here that examination of atomic and molecular lines toward the sight lines of DY Cen and HD 115842 shows that ionized lines are enhanced in strength in the sight line toward DY Cen relative to that of HD 115824 , which might suggest that the carrier(s) of the $6284 \AA$ and $7223 \AA$ DIBs could even have some contribution from ionized species.

Finally, we would like to remark that the very broad $4430 \AA$ DIB in DY Cen is almost identical to that in the comparison star HD 115842. This is shown in the right-hand panel of Figure 3 where we compare the 4400-4450 $\AA$ spectral region for both stars. The $4430 \AA \mathrm{DIB}$ has been linked to fullerenes bigger than $\mathrm{C}_{60}$ (e.g., $\mathrm{C}_{80}, \mathrm{C}_{240}, \mathrm{C}_{320}$, and $\mathrm{C}_{540}$ ) and/or buckyonions such as $\mathrm{C}_{60} @ \mathrm{C}_{240}$ and $\mathrm{C}_{60} @ \mathrm{C}_{240} @ \mathrm{C}_{540}$ (Iglesias-Groth 2007). Our finding also would be consistent with fullerenes and fullerenescontaining molecules not being especially overabundant toward DY Cen.

\section{CONCLUDING REMARKS}

Duley \& Hu (2012) show that the infrared features at $\sim 7.0$, $8.5,17.4$, and $18.8 \mu \mathrm{m}$ detected in astronomical sources with PAH-like-dominated IR spectra, such as those of the RCB stars DY Cen and V854 Cen (García-Hernández et al. 2011b), the reflection nebulae NGC 7023 and NGC 2023 (Sellgren et al. 2010), or the proto-PN IRAS $01005+7910$ (Zhang \& Kwok 2011) should be attributed to fullerene precursors (or protofullerenes) rather than to $\mathrm{C}_{60}$. Our non-detection of neutral $\mathrm{C}_{60}$ in DY Cen may support this claim. Thus, the carrier of the new $4000 \AA$ absorption band detected in DY Cen may be intimately related with the fullerene precursors seen at infrared wavelengths. Duley \& Hu (2012) suggest that fullerene precursors contain pentagonal carbon rings; the infrared emission features at $\sim 7.0$ and $16.4 \mu \mathrm{m}$ usually detected in objects with PAH-like-dominated IR spectra are characteristics of pentagonal rings (Moutou et al. 2000). Pentagonal carbon rings are present in carbon nanoparticles (e.g., HACs; Duley \& Hu 2012) and nanotubes although these have less perfect structures than $\mathrm{C}_{60}$ and other fullerenes.

In summary, the non-detection of neutral $\mathrm{C}_{60}$ in the highquality VLT/UVES DY Cen's spectrum may support recent experimental work, showing that the $\sim 7.0,8.5,17.4$, and $18.8 \mu \mathrm{m}$ IR features seen in sources with PAH-like-dominated spectra have to attributed to proto-fullerenes rather than to neutral $\mathrm{C}_{60}$. In addition, the new $4000 \AA$ DIB reported here (possibly also the carriers of the classical $6284 \AA$ and $7223 \AA$ DIBs) may be related to fullerene precursors; an organic compound containing pentagonal rings. These pentagonal carbon rings are usually present in HAC nanoparticles and nanotubes, suggesting that they may be intimately related with the formation process of fullerenes.

This work is based on observations obtained with the ESO Very Large Telescope at Paranal under the Director's Discretionary Time (DDT) program 284.D- 5048(A) (PI: D. A. GarcíaHernández). D.A.G.H. acknowledges support provided by the Spanish Ministry of Economy and Competitiveness under grant AYA-2011-27754. D.A.G.H. also thanks his son Mateo for his great patience during the realization of this work. D.L.L. also wishes to thank the Robert A. Welch Foundation of Houston, Texas for support through grant F-634.

Facilities: VLT:Kueyen

\section{REFERENCES}

Ballester, P., Modigliani, A., Boitquin, O., et al. 2000, Messenger, 101, 31 Braga, M., Larsson, S., Rosen, A., \& Volosov, A. 1991, A\&A, 245, 232 Cami, J., Bernard-Salas, J., Peeters, E., \& Malek, S. E. 2010, Science, 329, 1180 Cataldo, F., \& Iglesias-Groth, S. 2009, MNRAS, 400, 291

Cataldo, F., Strazzulla, G., \& Iglesias-Groth, S. 2009, MNRAS, 394, 615

De Marco, O., Clayton, G. C., Herwig, F., et al. 2002, AJ, 123, 3387

De Vries, M. S., Reihs, K., Wendt, H. R., et al. 1993, Geochim. Cosmochim. Acta, 57, 933

Duley, W. W., \& Hu, A. 2012, ApJ, 745, L11

Foing, B. H., \& Ehrenfreund, P. 1994, Nature, 369, 296

García-Hernández, D. A. 2012, in IAU Symp. 283, Planetary Nebulae: An Eye to the Future, ed. A. Manchado, L. Stanghellini, \& D. Schoenberner (Cambridge: Cambridge Univ. Press), 148

García-Hernández, D. A., Iglesias-Groth, S., Acosta-Pulido, J. A., et al. 2011a, ApJ, 737, L30

García-Hernández, D. A., Kameswara Rao, N., \& Lambert, D. L. 2011b, ApJ, 729,126

García-Hernández, D. A., Kameswara Rao, N., \& Lambert, D. L. 2011c, ApJ, 739,37

García-Hernández, D. A., Manchado, A., García-Lario, P., et al. 2010, ApJ, 724, L39

Goeres, A., \& Sedlmayr, E. 1992, A\&A, 265, 216

Herbig, G. H. 1995, ARA\&A, 33, 19

Herbig, G. H. 2000, ApJ, 542, 334

Hobbs, L. M., York, D. G., Snow, T. P., et al. 2008, ApJ, 680, 1256

Hunter, I., Smoker, J. V., Keenan, F. P., et al. 2006, MNRAS, 367, 1478

Iglesias-Groth, S. 2007, ApJ, 661, L167

Krelowski, J., Beletsky, Y., Galazutdinov, G. A., et al. 2010, ApJ, 714, L64

Kroto, H. W., Heath, J. R., Obrien, S. C., et al. 1985, Nature, 318, 162

Kwok, S. 2007, Physics and Chemistry of the Insterstellar Medium (Sausalito: Univ. Science Books)

Lambert, D. L., \& Rao, N. K. 1994, JA\&A, 15, 47

Leach, S. 1992, Chem. Phys., 160, 451

Luna, R., Cox, N. L. J., Satorre, M. A., et al. 2008, A\&A, 480, 133

Moutou, C., Verstraete, L., Léger, A., et al. 2000, A\&A, 354, L17

Sassara, A., Zerza, G., Chergui, M., Leach, S., et al. 2001, ApJ, 135, 263

Sellgren, K., Werner, M. W., Ingalls, J. G., et al. 2010, ApJ, 722, L54

Tull, R. G., MacQueen, P. J., Sneden, C., \& Lambert, D. L. 1995, PASP, 107, 251

Zhang, Y., \& Kwok, S. 2011, ApJ, 730, 126

Zhang, Y., Kwok, S., \& Hrivnak, B. J. 2010, ApJ, 725, 990 